\section{Hippocampal dysfunction associated with depression in temporal lobe epilepsy}

There is evidence to indicate that there is an increased prevalence of depression in individuals with temporal lobe epilepsy. Hippocampal abnormalities have been implicated in both temporal lobe epilepsy and depression. Hippocampal dysfunction might, therefore, explain the frequent co-occurrence of these conditions. To examine this hypothesis, 31 subjects who had experienced at least one complex partial or generalized tonic-clonic seizure in the 3 months prior to study start were evaluated using ${ }^{1} \mathrm{H}$-magnetic resonance spectroscopic imaging $\left({ }^{1} \mathrm{H}-\mathrm{MRSI}\right)$ of the hippocampus. The creatine/ $\mathrm{N}$-acetylaspartate ratio was used as a measure of ${ }^{1} \mathrm{H}-\mathrm{MRSI}$ abnormality. Depression symptoms were assessed using the Depression scale of the Profile of Mood States (POMS), and epilepsy-related factors and selfperceived social and vocational disability were also recorded.

Correlation analysis revealed a significant positive association between the extent of ${ }^{1} \mathrm{H}$-MRSI abnormality and severity of depression $(P<0.001)$. By contrast, no relationship was found between any of the seizure or selfperceived disability variables and POMS score. Linear regression demonstrated that $57 \%$ of the variance in depression symptoms could be explained by ${ }^{1} \mathrm{H}-\mathrm{MRSI}$ abnormalities. The authors conclude that hippocampal dysfunction is an important factor in the prevalence of depression in temporal lobe epilepsy, and call for further studies to clarify the role of the hippocampus in clinical depression.

Original article Gilliam FG (2007) Hippocampal ${ }^{1} \mathrm{H}$-MRSI correlates with severity of depression symptoms in temporal lobe epilepsy. Neurology 68: 364-368

\section{Antimyelin antibodies are not prognostic for progression to multiple sclerosis}

Patients presenting with a single neurologic event suggestive of multiple sclerosis (MS)known as a clinically isolated syndrome-are at high risk of progression to clinically definite MS, but outcome prediction for individual patients is difficult. A new study has tested the hypothesis that patients with serum antibodies against recombinant myelin oligodendrocyte glycoprotein (MOG) and purified myelin basic protein (MBP) are at an increased risk of progression.

Using a western blotting approach, Kuhle et al. measured serum anti-MOG IgG and antiMBP IgG and IgM antibodies in 462 patients. All patients analyzed in this study were enrolled in the multicenter BENEFIT trial designed to evaluate the effect of interferon $\beta 1 b$ on progression to MS in patients with a clinically isolated syndrome. During a median followup of 699 days, 150 patients were diagnosed with clinically definite MS and 331 with MS according to the McDonald criteria. On adjusted Cox proportional hazard analysis, no significant association was found between risk of clinically definite MS and the presence of anti-MOG or anti-MBP antibodies. Similarly, the risk of progression to MS according to the McDonald criteria was not significantly increased in any of the patient groups with positive IgM or IgG results. The variation in IgM and IgG status was not markedly different between individuals randomly assigned to receive placebo and those assigned interferon $\beta 1 \mathrm{~b}$.

The authors conclude that serum anti-MOG and anti-MBP antibodies, as detected by western blot, are not prognostic for progression to MS in patients with a clinically isolated syndrome.

Original article Kuhle J et al. (2007) Lack of association between antimyelin antibodies and progression to multiple sclerosis. N Engl J Med 356: 371-378

\section{'Benign' multiple sclerosis: difficult to characterize and predict clinical course}

Benign multiple sclerosis (MS) is usually defined as Expanded Disability Status Scale (EDSS) score $\leq 3$ ten years after disease onset. Patients with benign MS are often assured they are unlikely to experience as severe a disease course as other patients with MS. Long-term follow-up of patients diagnosed with benign MS in accordance with the standard definition, however, has now shown that patients with benign disease frequently experience disease progression and develop disability.

Sayao et al. studied 200 patients who had 'benign' disease approximately 10 years after disease onset. At 20 years, EDSS score had 